\title{
Zwei Umsetzungen neuerer literaturwissenschaftlicher Ansätze im Literaturunterricht
}

\author{
Jan Budňák
}

The paper compares two current perspectives of literary criticism - reader-response criticism and deconstruction - and gives an outline of their potentials in FLT. Results of two leading German researchers are applied in Czech school environment.

German - foreign language teaching - fiction - teaching literature - deconstruction - reader response

Der Beitrag vergleicht zwei neuere literaturwissenschaftliche Ansätze - die Rezeptionsästhetik und den Dekonstruktivismus - und ihre Anwendungsmöglichkeiten im DaF-Unterricht. Es werden Ergebnisse zweier dbz. Untersuchungen wiedergegeben und auf den Kontext des tschechischen Fremdsprachenunterrichts umgemünzt. Kernpunkt des Textes sind Überlegungen vom literaturdidaktischen Nutzen des poststrukturalistischen Verständnisses von Literatur.

Deutsch als Fremdsprache - Belletristik - Literaturdidaktik - Dekonstruktion Poststrukturalismus - Rezeptionsästhetik

Der vorliegende Beitrag verfährt größtenteils im Modus Bericht, Wiedergabe bzw. Kommentar. Er stellt zwei Beispiele für literaturdidaktische Unterrichts- bzw. Aufgabenentwürfe vor, die von zwei unterschiedlichen literaturwissenschaftlichen Ansätzen ihren Ausgang nehmen und kommentiert sie im Hinblick auf ihre Anwendbarkeit im DaF-Unterricht. Es handelt sich erstens um das Modell, das von Swantje Ehlers in ihrer 1992 erschienenen Fernstudieneinheit „Lesen und Verstehen“ erarbeitet wurde, zweitens um die literaturdidaktischen Arbeiten von Clemens Kammler. Den hier wiedergegebenen unterrichtspraktischen Konzepten ist u. a. gemeinsam, dass der jeweilige theoretische Bezugsrahmen der Konstanzer Wirkungsästhetik(WolfgangIser) beiEhlersbzw.desfranzösischenPoststrukturalismus (Michel Foucault) bei Kammler zwar kenntlich bleibt und als Fundgrube für eigenständige Unterrichtsentfaltung dient, aber beide Literaturdidaktiker verzichten nicht im Geringsten auf die Basis der bewährten hermeneutischen Textanalyse. Es kann als positiv gelten, dass im Literaturunterricht ein gewisser „methodischer Eklektizismus“" (hinsichtlich der literaturwissenschaftlichen Interpretationsmodelle) nicht verpönt wird. Der Verfasser des „poststrukturalistischen“ Kapitels in den Grundzügen der Literaturdidaktik, Jürgen Förster, hält die in der Literaturdidaktik betriebene, pragmatisch geleitete „Versöhnung“ der literaturwissenschaftlichen „Interpretationsschulen“ sogar für eines ihrer größten Verdienste („Differenzen zwischen hermeneutischen und poststrukturalistischen Modellen brauchen dabei nicht Irritation auszulösen, sondern sind als Chance zu sehen, diese im Unterricht 
produktiv zu machen“ FÖRSTER: 2002, 241). Dennoch unterscheiden sich die Strategien der rezeptionsästhetisch bzw. poststrukturalistisch inspirierten Literaturdidaktik in Bezug aufText, Lerner und Unterrichtsziel in vielen wesentlichen Punkten. Das soll im Folgenden die Beschreibung der Konzepte von Ehlers und Kammler verdeutlichen.

\section{Wirkungsästhetisch inspirierte Literaturdidaktik}

Swantje Ehlers versucht in ihrer Monographie Lesen als Verstehen. Zum Verstehen fremdsprachlicher literarischer Texte und zu ihrer Didaktik eine ideenreiche und unterrichtsgerechte Synthese von hermeneutischen und rezeptionsästhetischen Verfahren. Die Arbeit richtet sich an (fremdsprachliche) Leser, die sich literarische Texte erschließen wollen. Ehlers' Schlüsselbegriffe werden wie folgt formuliert: „[...] Leser und sein Verstehen. Das Gespräch zwischen Text und Leser ist das Thema, das sich durch die Studieneinheit zieht. Die Faktoren, die dieses Gespräch beeinflussen, werden in den einzelnen Kapiteln herausgearbeitet und auf ihre lesedidaktische Bedeutung hin geprüft" (EHLERS: 1992, 4).

Wie schon angedeutet, gibt Ehlers literaturwissenschaftlich betrachtet die hermeneutische „Sinn-Suche“ nicht auf, obwohl sie den eigenen Standpunkt explizit „zur Diskussion stellt und sich dem Gespräch mit den Lesern öffnet“ (EHLERS: 1992, 5). Die Forschungsliteratur sowie die Unterrichtspraxis bieten eine Fülle von Beispielen dafür, wie entmutigend die Folgen einer engherzig "fingierten“ oder wiederum einer uferlos assoziativen „Sinnsuche“ für die Schüler sein können. Der letzteren, bei der Wirkungsästhetik viel unmittelbarer drohenden Gefahr einer nicht am Text korrigierten Deutungssubjektivität entgeht Ehlers, indem sie nah am Text arbeitet: Die Studieneinheit möchte „deutlich machen, dass literarische Strategien und Darstellungstechniken konventionalisiert sind und dass ein Leser diese Konventionen kennen muss. [Die Studieneinheit, JB] legt auch Wert darauf klarzumachen, dass das Verstehen von Texten nicht beliebig ist, sondern dass man die ihnen eigene Sprache kennen muss, um mit ihnen ,sprechen' zu können“ (EHLERS: 1992, 4). Dieses textanalytische Handwerk kommt dann auch im unterrichtspraktischen Teil nicht zu kurz: „Strukturelle“ Aufgaben wie „Wer sagt ...? Was ... ? Wie ...? Worüber ...?" (EHLERS: 1992, 9), aber auch Fragen nach der Perspektive des Erzählers (EHLERS: 1992, 16 f.) oder der Zeitstruktur des Erzählten (EHLERS: 1992, 20) werden nicht ausgeblendet. Fast rein strukturalistisch ist dann das Kapitel „Ästhetische Wahrnehmung“ (EHLERS: 1992, 42-49), das vorwiegend stilistische Unterschiede zwischen einer Grimmschen Märchen-Vorlage und der Neufassung von Günter Kunert erarbeitet.

Allerdings sind Ehlers' Schwerpunkte eindeutig lesedidaktisch und nicht textwissenschaftlich. Auf der literaturdidaktischen Ebene zeigt sich, wie gewinnbringend gerade die Koordinierung eines strukturalistisch-hermeneutischen 
Rahmens („konventionalisierte Darstellungstechniken“) mit einer individualisierendwirkungsästhetischen „Füllung“ ist. Allein das dem Buch beigefügte, recht knappe Glossar macht die rezeptionsästhetische Denkrichtung klar. Neben eher „literaturwissenschaftlichen“ Einträgen (,impliziter Leser“, „Rezeptionsästhetik“) werden dort vor allem Begriffe vorgestellt, die die Lektüre von literarischen Texten individualisieren oder als Aufforderung zur Handlung verstehen (,antizipieren“, „Leseprotokoll“, „projizieren“, „aktivieren“, „Applikation“, vgl. EHLERS: 1992, $108 \mathrm{f.}$.).

Ähnlich beschaffen sind auch die konkreten Aufgaben, die Ehlers für die Unterrichtsarbeit mit literarischen Texten vorstellt. Sie wählt vor allem kürzere, zeitgenössische, situativ anschauliche Texte, in denen die Schüler allerdings oft eine provozierende „Inkohärenz“ (vgl. EHLERS: 1992, 19), eine „Unbestimmtheitsstelle“ (Wolfgang Iser) auf der Textoberfläche „überbrücken“ müssen. Sie müssen also den Sprung vom wörtlichen Verständnis zum Textverständnis schaffen, wozu ihnen zahlreiche Hilfestellungen gegeben werden. Verstehen in diesem Sinn ist dann wirklich eine Handlung. Sie kann entweder spontan verlaufen, wie bei Ehlers" erstem Text „Verstehen“, dessen Erfassen großteils auf textexterne „Weltkenntnis“ baut. ${ }^{1}$ Das spontane Verständnis wird dann im Lektüre-Nachfeld versprachlicht und didaktisch aufgearbeitet.

Gleichzeitig werden die Schüler mit vielen Impulsen zum „Sich-ins-GeleseneEinbringen“"versorgt. Gerade in den vielfältigen Aufgaben dieser Art besteht wohl die größte Wirksamkeit von Ehlers' rezeptionsästhetischem Ansatz. Erstens wird den Schülern schon zu Beginn die Abhängigkeit verschiedener "Spontanverständnisse“ von den Positionen der „Interpreten“ vor Augen geführt. Die Schüler werden mit unterschiedlichen Sichtweisen auf einen Text konfrontiert und sollen von den Schwerpunkten der jeweiligen Interpretation auf den Background des jeweiligen, zur Auswahl stehenden Interpreten schließen. Des weiteren werden vor der Lektüre Erwartungen gesammelt und verglichen, es werden aufgrund von Teilangaben und Teilergebnissen unterschiedlicher Art Prädiktionen zu Figurenoder Handlungsentwicklung gemacht, es werden Zeit-, Raum- oder Temposprünge entdeckt und auf deren Wirkung hin besprochen, es wird nach Parallelen und Aktualisierungen gesucht. Kurz: Der Leser befindet sich - Ehlers' theoretischer Linie nach - in einem ständigen Gespräch mit dem Text. ${ }^{2}$

1 „Verstehen“

Du bist noch zu klein, um das zu verstehen,/ das kannst du noch nicht verstehen,/ nein, das verstehst du nicht,/ versteht das nicht,/ noch nicht,/ verstanden!!! (Hans Manz, zitiert nach EHLERS: 1992, 6).

2 Vgl. Ehlers` Kapitelüberschriften „Der Leser als Mitspieler“, „Lesen als Tätigkeit“, „Das Gespräch mit einem fremdsprachlichen Text". 
In Ehlers' individualisierender Herangehensweise liegt außerdem die interkulturelle Lesart wie die Nuss in der Schale. Im Einklang mit der wirkungsästhetischen Linie wurde sievor allem von dem Münchner DaF-Professor Dietrich Krusche (KRUSCHE: 1985) ausgearbeitet (Literatur und Fremde. Zur Hermeneutik kulturräumlicher Distanz). Ein Dialog „Zwischen Kulturen“ wird in Ehlers' Unterrichtsentwürfen nicht primär zwischen Sprechern von zwei Sprachen geführt, sondern z. B. (durch Vermittlung des literarischen Textes) zwischen zwei Altersgruppen, zwei historischen Epochen oder zwei Weltanschauungen. Das Einnehmende der rezeptionsästhetischen Textdidaktik wird auch hier gewahrt.

\section{Poststrukturalistische Literaturdidaktik}

Während Ehlers' wirkungsästhetischeLiteraturdidaktik das ganze Wissen und Können der Schüler für das Textverständnis aktivieren will, stellt der poststrukturalistische/ dekonstruktivistische Literaturunterricht sie eher in eine prinzipiell prüfende Position gegenüber dem Text und dem eigenen Eindruck davon. Während Ehlers' Leser „konventionalisierte literarische Strategien und Darstellungstechniken erkennen müssen“, um nicht eine der Wurzeln des Textverständnisses zu verlieren, suchen Clemens Kammlers Schüler nach Widersprüchen zwischen konventionalisierter Struktur und anderen Elementen des Textes. Während Ehlers einzelne Perspektiven von Lehrer und Schülern „zusammenträgt“, lässt Kammler meistens Schülergruppen an einander widersprechenden Deutungen von Text bzw. Kommentar arbeiten, die dann auf unterschiedlichen Ebenen konfrontiert werden. Der Vorschlag, das dekonstruktivistische Prinzip als Grundlage der Unterrichtsorganisation zu nutzen, klingt sehr spannend. Das wohlbekannte Vorurteil gegenüber der poststrukturalistischen Literaturwissenschaft lautet allerdings (ähnlich wie bei der Rezeptionsästhetik), sie zerstöre im Sinne des postmodernen ,anything goes“ alle erdenklichen Interpretationen eines literarischen Textes. Der Leser würde am Ende verzweifelt mit leeren Händen dastehen. Eine solche Perspektive wäre selbstverständlich für die Literaturdidaktik noch weniger geeignet als für die Literaturwissenschaft.

Erfreulicherweise trifft dieses Vorurteil auf die meisten konkreten literaturdidaktischen Unterrichtsvorschläge, die an dekonstruktivistische Ansätze anknüpfen, nicht im Geringsten zu. Als Beispiele will ich hier einige Arbeiten von Clemens Kammler heranziehen, die vor allem von den Ideen von Michel Foucault ihren Ausgang nehmen. ${ }^{1}$ Kammler formuliert das Ziel seiner Unterrichtsentwürfe als „Dekonstruktion homogener Sinn-Entwürfe“ (KAMMLER: 2000, 45), die über einen Teil der Bedeutungen, die der Text enthält, beim Interpretationsverfahren "gestülpt“ werden. Die Gründe, die zu dieser Einengung führen, können

1 Kammler ist u. a. Autor einer Foucault-Monographie (KAMMLER: 1986). 
unterschiedlicher Natur sein. Am wenigsten fassbar, aber gleichzeitig wohl am wirksamsten, ist das Bedürfnis von Lesern, einen literarischen Text „auf einen Punkt" zu bringen. Das betrifft sowohl viele Fachleute, z. B. Autoren von Literaturgeschichten oder Unterrichtshilfen, als auch viele Literaturlehrer, -schüler und -studierende. An tschechischen Bildungsinstitutionen dürfte diese Tendenz noch stärker sein, da hier der Textlektüre eher die Textwiedergabe vorgezogen wird. So natürlich dieses vereinheitlichende Bedürfnis auch sein mag: Gerade in der von kaum wohlmeinenden Überzeugungsstrategien überfüllten Gegenwart wäre eine kritische Einstellung zum Lesen wünschenswert. Die Lektüre von literarischen Texten sollte gerade die Bruchstellen in scheinbar homogenen Textbedeutungen bzw. schon existierenden Interpretationen anpeilen, da dadurch ein prinzipiell prüfender Umgang mit komplexen (Lebens-)Situationen geübt wird. Auch das oder vor allem das - wäre im Hinblick auf kulturelle Inhalte unter Lernerautonomie zu verstehen.

\subsection{Kafka - Arbeit am Mehrdeutigen}

Der poststrukturalistische literaturdidaktische Text deutschsprachiger Provenienz, der Karriere gemacht hat, ist wohl Clemens Kammlers „Neue Literaturtheorien und Unterrichtspraxis. Eine Untersuchung am Beispiel von Kafkas, Vor dem Gesetz “" von 1993. Er wurde als literaturdidaktischer Nachsatz im Sammelband Neue Literaturtheorien in der Praxis veröffentlicht, dessen Aufgabe es war, „Bewährungsproben“ neuer Literaturtheorien an einem literarischen Text zu liefern. Für die Erprobung unterschiedlicher Lesarten wurde Kafkas „Türhüterlegende“ aus dem „Prozess“ gewählt, ein Text, der vieles zuzulassen scheint, aber auf den zweiten Blick vielleicht noch mehr ausschließt.

Clemens Kammler weist in seinem Beitrag zunächst auf die zahlreichen Risiken einer - nicht nur schulischen - Kafka-Lektüre hin, die sich vielleicht unter das Stichwort „Begrenzung der Vieldeutigkeit“ zusammenfassen ließen. Im eigenen Unterrichtsvorschlag geht es ihm dann logischerweise um die Erarbeitung der Bedeutungsvielfalt des Textes. Die Rezeption der Schüler soll zunächst ungesteuert verlaufen, so dass sie die Warum-Fragen an den Text selbst stellen: „Warum gelingt es dem Mann vom Lande nicht, in das Gesetz zu gelangen? Was ist dieses Gesetz überhaupt?" (KAMMLER: 1993, 200). Genauso wie Ehlers fährt Kammler auch fort: „In dieser Phase ist ein hermeneutischer Umgang mit dem Text notwendig, müssen alle Deutungsansätze der Schüler ernstgenommen und thematisiert werden “ (KAMMLER: 1993, 200). Schon an diesem Punkt, berichtet Kammler weiter, wird wohl eine Diskussion entstehen, ob das Scheitern des Menschen vor dem Gesetz „seinem eigenen Versagen oder der Übermacht des Türhüters zuzuschreiben sei“ (KAMMLER: 1993, 200). Die Feststellung der paradoxen Situation, die beide Antworten zugleich anbietet und entkräftet, sei in dieser noch „spontanen“ Phase 
der Textbesprechung genug: Mögliche Interpretationen folgen im Anschluss an erweiternde Lektüre, etwa von dem „Brief an den Vater“ oder der „Verwandlung“. Mit oder ohne diese weiterführende Lektüre gelangen die Schüler aber wie von selbst zu der dekonstruktivistischen Schlussfolgerung, die das Problem der (eindeutigen) Interpretierbarkeit von Text und Welt thematisiert. Kammler ist dabei um positive Ergebnisse des Literaturunterrichts bemüht: „Kafkaesk wird Unterricht dann, wenn er sich darauf beschränkt, die Unlösbarkeit der Rätsel zu konstatieren, statt darüber hinaus die Funktionsweise der Machverhältnisse erfahrbar zu machen und zu analysieren" (KAMMLER: 1993, 203).

So motivierend der eben wiedergegebene Unterrichtsvorschlag auch sein mag, er bestärkt einen weiteren Einwand gegen eine Literaturdidaktik, die auf dekonstruktivistischen Prinzipien aufbaut. Ließe sich diese Art von Beschäftigung überhaupt mit dem literarischen Text, die "gleich-berechtigte" Lesarten gegeneinander ausspielt und so zu prüfender Lektüre, fruchtbarer Diskussion und Hinterfragung von vorgefertigten Sinnmustern führt, auch etwa bei Fontane oder Lessing betreiben? Wer sträubt sich schon gegen eine eindeutige Interpretation so eindeutig wie Kafka?

\subsection{Büchner - Arbeit am Fragment}

Dass Schüler und Studierende nicht nur den ambivalenten Kafka „dekonstruieren“ müssen, sondern sich auch an scheinbar weniger befremdenden Texten dekonstruktivistisch versuchen können, zeigt Clemens Kammlers Unterrichtsentwurf zu Georg Büchners „Woyzeck“ (KAMMLER: 2000, 41-54). Das auslösende Moment der Unterrichtsplanung zu diesem Drama ist die Tatsache, dass es sich hier eigentlich nicht um einen "definitiven“ Text handelt, sondern um ein Fragment. Während die erste Hälfte der Szenen eine relativ wenig umstrittene Reihenfolge hat, wurde fast jede der Szenen nach der Ermordung Maries durch Woyzeck als Schlussszene für jeweils alternative Fassungen gewählt. Mit einer so tief greifenden Schwerpunktsetzung, wie es die Bestimmung des Dramenschlusses ist, muss aber in der Folge auch das Vorfeld harmonisiert werden.

Bei dieser Überlegung setzt Kammler an. Er entdeckt den springenden Punkt des Textes, von dem die Entscheidung über den Abschluss des Stückes am ehesten abhängig sein wird: Die Tötung der untreuen Lebensgefährtin durch den verwirrten Soldaten Woyzeck. Ist der Mord aber auf Woyzecks Misshandlung durch seine Vorgesetzten zurückzuführen, oder gerät er in geistige Umnachtung aufgrund der gerade entdeckten Untreue seiner Lebensgefährtin? Wird das Drama also sozialkritisch oder psychologisch interpretiert? Ist Woyzeck Opfer eines inhumanen Gesellschaftssystems oder seiner charakterpsychologischen Anlage?

Kammler entwirft eine umfangreiche Aufgabensequenz, die die Schüler zu einer eindeutigen Entscheidung für die eine oder andere Variante bringt. In der ersten 
Stunde wird den Schülern der Text der Ermordungsszene und das Personenregister des Dramas vorgelegt. Sie werden dazu aufgefordert, eigene Handlungsentwürfe zu produzieren. Diese Handlungsskizzen laufen aufgrund des zur Verfügung stehenden Materials höchst wahrscheinlich auf eines der folgenden beiden Motive für den Mord hinaus: Eifersucht oder die soziale Drangsalierung Woyzecks. Die beiden verschiedenen Sichtweisen ergeben sich dabei allein aus der Figur des Tambourmajors bzw. der Gegenüberstellung von Woyzeck mit dem Hauptmann und dem gelehrten Doktor.

Der Startpunkt der dekonstruktivistischen Lesart des Dramas besteht darin, dass sich für beide Handlungsmotive im Drama starke Evidenz finden lässt. Diese kreative „Divergenz“ wird durch den nächsten Schritt des Unterrichtsentwurfs noch verstärkt, nämlich in Form eines Fragebogens, der die Eindrücke der Schüler von der Lektüre des Dramas festhält. Bei einem Unterrichtsversuch, bei dem die Schüler einen solchen Fragebogen ausfüllten, hat sich gezeigt, dass bei denjenigen Fragen, bei denen nach interessanten bzw. unverständlichen Stellen/Szenen gefragt wurde, die Antworten unterschiedlich ausfielen, und zwar je nachdem, welche Szenen mit der von ihnen erarbeiteten Handlungsskizzen vereinbar waren. „Die Schüler erkannten im Verlauf dieser Auseinandersetzung, [...] dass ihre jeweiligen Interpretationshypothesen also in einem wechselseitigen Bedingungsverhältnis zu dem Verstehen bzw. Nichtverstehen einzelner Szenen standen. So hatten diejenigen, die den Hauptakzent bei einer rein ,psychologischen' Problematik vermuteten, jenen Szenen die größte Bedeutung beigemessen, die Woyzecks Eifersucht verstehbar machen, andere Szenen demgegenüber als unwichtig bzw. dysfunktional angesehen usw." (KAMMLER: 2000, 47). Kammler führt auch das Urteil eines Schülers an, der die überraschende „Regel der Interpretation“ treffend zusammenfasst: „Eigentlich haben die meisten im ,Woyzeck' das gefunden, was sie darin gesucht haben" (KAMMLER: 2000, 47). Diese Erkenntnis wertet Kammler als das „Stadium der Dekonstruktion“ aus: Die Schüler haben ,ihre Sinnkonstrukte in Bezug auf den Text nicht nur formuliert, [...] sondern sie zeigten die Bereitschaft, ihre Sinnentwürfe [...] im Dialog mit dem Anderen in Frage zu stellen“ (KAMMLER: 2000, 48).

Wie fruchtbar eine auf diese Weise vorgeprägte Arbeitsstimmung im Literaturunterricht werden kann, zeigen die Ergebnisse der anschließenden Aufgaben. Auf dieser Stufe werden zwar die klassischen hermeneutischen Analyseverfahren wie die Untersuchung von Handlungsstruktur oder Figurenkonstellation nicht vernachlässigt, aber sie können nun alle auf das Grunddilemma bezogen werden. So wird etwa die Figurenanalyse so erarbeitet, dass das Agieren der Figuren im Privatbzw. Arbeitsbereich („psychologische“ versus ,gesellschaftsbedingte“ Motivation) als ein weiterer Aspekt zu den schon vorhandenen Interpretationen hinzugefügt werden kann.

Zwei Aktivitäten stellen bei Kammler den Höhepunkt der prüfenden Lektüre des Dramas dar, wobei die erste von den Schülern selbst als Vorschlag kam. Die 
Schüler wollten sich als „Herausgeber“ des Woyzeck-Fragments versuchen und selbst die Reihenfolge der letzten sieben Szenen des Dramas im Einklang mit dem jeweiligen inhaltlichen Schwerpunkt vorschlagen. Durch Unterschiede in der Anordnung der Schlusssequenz sind Lösungsvarianten entstanden, die die unterschiedlichen Interpretationsstränge noch einmal konkretisiert haben. Die Gerichtsdienerszene am Schluss legt z. B. nahe, dass Woyzecks Wahnsinn Folge seiner sozialen Isolation ist; die symbolische "Teichszene“ ist ein Hinweis auf die Möglichkeit von Woyzecks „Reinigung“; eine weitere Szene (die Szene „Eine Person ertrinkt") enthält einen möglichen Hinweis auf Woyzecks Selbstmord, woraus sich zumindest andeutungsweise eine andere Szenenanordnung ergeben würde. Kammler berichtet auch, dass diese Aktivität von den Schülern als besonders ertragreich gesehen wurde, musste doch die Berechtigung der einzelnen Vorschläge sorgfältig begründet werden.

Die letzte Phase der Unterrichtsreihe zu Büchners „Woyzeck“ ist theaterbezogen und schließt eine weitere Herausforderung an „definitive" Lesarten ein. Kammlers Schüler trafen sich mit dem Regisseur R. Karge, der den "Woyzeck“ gerade in einer neuartigen Inszenierung auf die Bühne brachte. Seine Lesart des Dramas hat eine Konstante der Woyzeck-Deutung in Frage gestellt: Woyzeck wird nicht als Opfer seiner seelischen bzw. sozialen Anlage porträtiert, sondern als aktiver Widerständler gegen seine „Feinde“. Die Schüler stellen zunächst fest, dass der Regisseur ihnen keine konkreten Erklärungen dafür geben kann, wie man mit den fragwürdigen Folgen dieser Sichtweise fertig werden kann. Allerdings werden - im Einklang mit Kammlers Intention - die Textdeutungsprozesse einer nochmaligen Überprüfen ausgesetzt und diesmal eher bestätigt als entwertet: Woyzeck als einen tatkräftigen Kämpfer zu deuten ist zwar attraktiv, aber es stellt den Leser vor kaum erklärbare Widersprüche mit dem Dramentext. So werden die Schüler auch mit einer freieren Adaptation konfrontiert und sind im Stande, ihre Distanz vom Text zu begründen. Diesen Ertrag hätte man gerade beim postrukturalistischen Literaturunterricht am wenigsten erwartet.

Werden in Kammlers Kafka-Unterricht verabsolutierte Teilinterpretationen dekonstruiert und bei seinem Büchner-Unterricht die durch den Herausgeber begünstigten Bedeutungen hinterfragt, bietet sich bei der Behandlung des „Klassikers unter Klassikern“ - Goethes Faust - die Dekonstruktion des „Phänomens Goethes Faust“ an. Das, was Kammler in der poststrukturalistischen Begrifflichkeit „Diskurskritik“ bzw. „diskurskritische Unterrichtsvorschläge“ nennt, ist eine didaktisch sehr spannende Gegenüberstellung von Text einerseits und Kommentar bzw. Populärbild andererseits. Ähnliches unternimmt Kammler mit Texten von Heinrich Mann und Heiner Müller, mit Texten zur Shoa oder solchen aus der Gegenwartsliteratur. Spätestens beim Erscheinen des von Kammler und Bogdal herausgegebenen Buches (K)ein Kanon. 30 Schulklassiker neu gelesen 
(2000) sollte klar sein, dass der poststrukturalistische Ansatz nicht nur für die Behandlung besonders verzwickter oder „belasteter“ Texte taugt, sondern dass jeder literarische Text (im Schulkanon) a priori und zunehmend verzerrenden SinnVerarbeitungsprozessen ausgesetzt ist und damit für Dekonstruktion Stoff genug bietet. Am Ende steht etwas da, was in jedem Literaturunterricht (sei es in der Mutter- oder Fremdsprache) angestrebt werden sollte: gezielte Auseinandersetzung mit verabsolutierten Einseitigkeiten bzw. mit ideologisierten Verunglimpfungen. So kann Literaturdidaktik gerade auf Bedürfnisse antworten, die sich aus den Forderungen der modernen Fremdsprachendidaktik ergeben: Lesekompetenz und Lernerautonomie.

\section{Literatur:}

EHLERS (1992): Ehlers, Swantje. Lesen als Verstehen. Zum Verstehen fremdsprachlicher Texte und zu ihrer Didaktik. Berlin: Langenscheidt, 1992.

FÖRSTER (2002): Förster, Jürgen. Analyse und Interpretation. Hermeneutik und poststrukturalische Tendenzen, in: Bogdal, Klaus-Michael (Hg.): Grundzüge der Literaturdidaktik. München: Deutscher Taschenbuch Verlag, 2002, 231-246.

KAMMLER (1986): Kammler, Clemens. Michel Foucault. Eine kritische Analyse seines Werkes. Bonn: Bouvier, 1986.

KAMMLER (1993): Kammler, Clemens. Neue Literaturtheorien und Unterrichtspraxis. Eine Untersuchung am Beispiel von Kafkas „Vor dem Gesetz“, in: Bogdal, Klaus Michael (Hg.): Neue Literaturtheorien in der Praxis. Textanalysen von Kafkas "Vor dem Gesetz". Opladen: Westdeutscher Verlag, 1993, 187-206.

KAMMLER (2000): Kammler, Clemens. Neue Literaturtheorien und Unterrichtspraxis. Positionen und Modelle. Hohengehren: Schneider Verlag, 2000.

KRUSCHE (1985): Krusche, Dietrich. Literatur und Fremde. Zur Hermeneutik kulturräumlicher Distanz. München: Iudicium, 1985. 